\title{
Primary breast MALT lymphoma: a case report
}

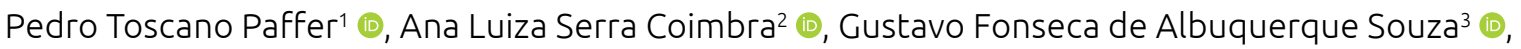

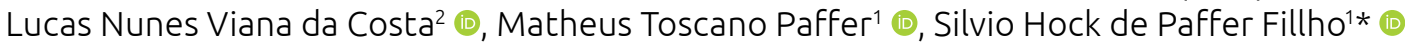

\section{ABSTRACT}

A 42-year-old woman, with no history of autoimmune diseases or risk factors for cancer, sought a private medical clinic for undergoing breast imaging tests, noticing the presence of a solid nodule with indistinct margins - BI-RADS 4 - in the left breast. An ultrasound-guided core biopsy was performed and complemented by histopathological and immunohistochemical studies, confirming the diagnosis of primary small B-cell MALT lymphoma. After treatment with radiotherapy, the patient evolved with remission, maintaining annual follow-up with a specialist physician. The importance of routine screening for pathologies that affect the breasts is highlighted, aiming at their early diagnosis. In addition, radiotherapy has good prognostic results at the expense of surgical treatment.

KEYWORDS: lymphoma, B-cell. marginal zone; breast neoplasms; radiotherapy.

\section{INTRODUCTION}

Primary breast lymphoma (PBL) is a rare manifestation of breast cancer, accounting for $0.4 \%-0.5 \%$ of all malignant breast lesions ${ }^{1}$. Despite presenting clinical characteristics of other types of breast cancer, PBL occurs without evidence of systemic disease ${ }^{2}$. In addition, it is characterized by the presence of breast tissue associated with lymphocytic infiltrate and by the presence of ipsilateral axillary lymphadenopathy of the primary lesion ${ }^{3}$.

The most common type of PBL is the non-Hodgkin diffuse large B-cell lymphoma, accounting for $50 \%$ of all PBL cases and $2 \%$ of all extranodal lymphomas. About $15 \%$ of PBL are of the follicular subtype; $12 \%$, of the mucosa-associated lymphoid tissue lymphoma (MALT) type; and 16\%, of Burkitt and Burkittlike lymphoma type 4

The mean age at PBL diagnosis is 68 years, being prevalent in women, which suggests a relation with the female hormone estrogen. Patients may present with symptoms such as local pain or inflammation, palpable lymph nodes, or painless masses in the outer quadrant of the affected breast; however, asymptomatic cases are more prevalent. In these asymptomatic cases, the diagnostic suspicion is evidenced after undertaking a mammogram test showing incidental findings such as noncalcified soft solitary masses ${ }^{5}$.
After performing fine-needle aspiration, or core biopsy, for histopathological analysis, the diagnosis can be confirmed, requiring an active search for primary sites of cancer, especially in the gastrointestinal tract (GIT). Computed tomography (CT) or magnetic resonance imaging (MRI) are performed to rule out metastases and confirm the primary site ${ }^{6}$.

Surgical treatment has been losing ground in the scientific community, considering that it does not show benefits when compared with radiotherapy or chemotherapy. Therefore, for localized cases of MALT lymphoma, treatment based on radiotherapy is indicated, whereas for more advanced cases, radiotherapy is adopted in combination with chemotherapy. Additional predictive factors for the disease staging include age, numbers of extranodal sites, course of treatment, and levels of lactate dehydrogenase $(\mathrm{LDH})^{7}$.

The present study aimed to report the case of an unusual presentation of oncological disease, a primary breast MALT lymphoma, seeking to evidence the importance of adequate followup for the patient's good prognosis.

\section{CASE REPORT}

This is a case report of a woman, aged 42 years, white, with no medical history of interest, with a G2P2A0 obstetric history, who

${ }^{1}$ Faculdade de Medicina de Olinda - Olinda (PE), Brazil.

2Universidade de Pernambuco - Recife (PE), Brazil.

${ }^{3}$ Universidade Católica de Pernambuco - Recife (PE), Brazil.

*Corresponding author: silviopaffer@gmail.com

Conflict of interests: nothing to declare.

Received on: 06/29/2021. Accepted on: 02/08/2021. 
gave birth for the last time 14 years ago, in addition to denying smoking or other risk factors for oncological diseases. The patient sought a private medical service for routine mammography. Heterogeneously dense breast tissue and the presence of normal lymph nodes in the left axillary extension were observed; no nodular images were detected due to the high density of the breast tissue, and the examination was complemented by breast ultrasound for comparative study.

According to the ultrasound results, there was a solid, hypoechoic nodule with regular contours, located in the right breast at 1-o'clock position, measuring $0.8 \times 0.4 \times 0.8 \mathrm{~cm}, 1.2 \mathrm{~cm}$ from its center to the skin. A solid, hypoechoic nodule with indistinct margins was also observed, located in the left breast at $1 / 2$ o'clock position, measuring $2.0 \times 0.8 \times 1.8 \mathrm{~cm}, 1.2 \mathrm{~cm}$ from its center to the skin. After comparing mammography and ultrasound tests, the patient was classified as BI-RADS 4 and proceeded to diagnostic investigation, undergoing an ultrasound-guided core biopsy two days later. Four fragments were removed with a 14-gauge needle, from both lesions, for anatomopathological study; the right breast nodule being classified as intra- and pericanalicular fibroadenoma (Figure 1).

On the left breast, lymphocyte proliferation was observed, including sparse reactive follicles, with a predominance of mature lymphocytes, mainly affecting the periductal stroma, intraepithelial lymphocytes (Figure 2).

With the differential diagnosis proposed by the pathologist between chronic mastitis and low-grade lymphoma, an immunohistochemical study was carried out.

According to immunohistochemical examination, the presence of a dense lymphocytic infiltrate in the breast parenchyma was verified, consisting of small atypical B cells (CD20+), several lymphoid follicles with prominent germinal centers, and numerous foci of lymphoepithelial lesions that are highlighted by immunostaining for cytokeratin and CD20. A low cell proliferation

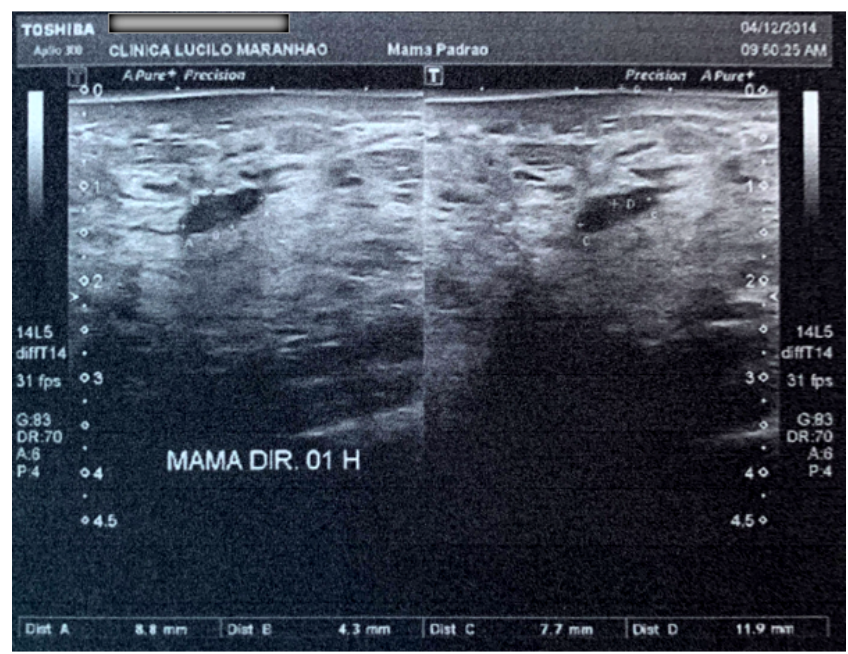

Figure 1. Right breast nodule. index was also observed in the monocytoid lymphoid population. The set of findings elucidated the diagnosis of primary small B-cell MALT lymphoma of the breast. B cells were positive for CD20 and CD10, but negative for CD3 (Table 1).

Upon diagnosis, the patient underwent positron emission tomography (PET-CT) to investigate additional lesions, in which no additional mass or lymphadenopathies were observed (Figure 3).

After staging of the lesion and deciding on the appropriate therapy, the patient started radiotherapy treatment with a total dose of $4140 \mathrm{cGy}$ during 23 sessions, with no complications. Nowadays, about five years after the initial investigation, the patient is in remission carrying out annual follow-up with a specialist physician, with no new neoplasms or metastases having appeared.

\section{DISCUSSION}

MALT lymphomas are indolent extranodal neoplasms that can be manifested in a wide variety of organs, including stomach, large or small intestine, lungs, salivary glands, thyroid, skin, thymus, tonsils, liver, kidney, bladder, and breast. They represent a subset of low-grade B-cell lymphomas and account for 7\%-8\% of all types of B-cell lymphomas and $12 \%$ of all PBL. The breast involvement of adjacent lymph nodes can be explained by the origin of the MALT from the mammary ducts and lobules, in addition to the intramammary lymph nodes ${ }^{4,8}$.

Once in the breast, these lymphomas mainly affect women with an average age of 68 years (47-92), being especially observed during pregnancy or postpartum, and may be related to personal antecedents such as autoimmune diseases. However, this does not apply to the case in question, as the patient was diagnosed with the disease at 42 years of age and had no history of autoimmune diseases or recent pregnancy. Usually, the neoplasm presents itself as a large unilateral and painless mass, rarely bilateral, and with an average size of $3 \mathrm{~cm}^{9,10}$.

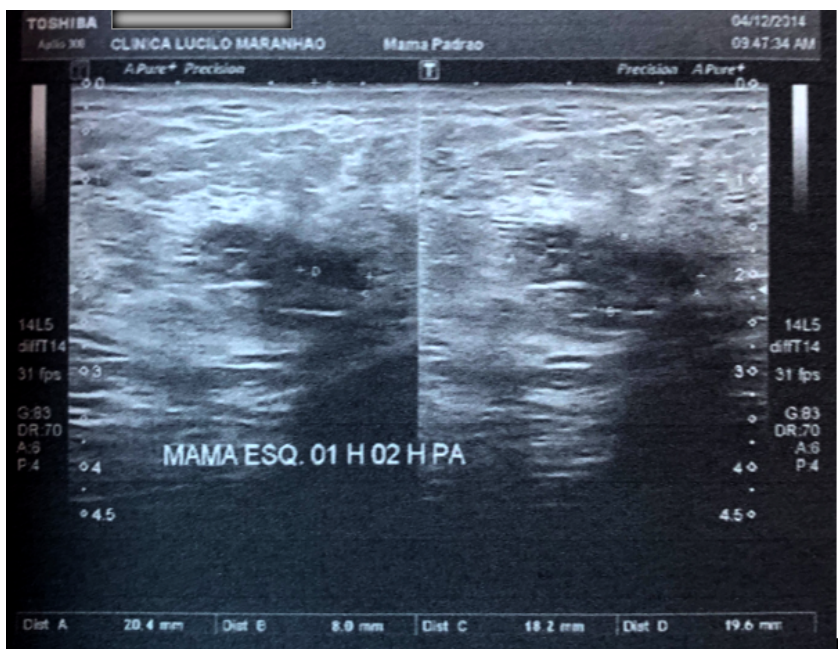

Figure 2. Left breast nodule. 
Considering the clinical characteristics, the patient remains in accordance with the literature, being asymptomatic, including without presenting the classic B symptoms - systemic symptoms of fever, night sweat, and unexplained loss of more than $10 \%$ of weight in six months -, being the tumor identified as an incidental finding in imaging tests ${ }^{1,9,10}$.

The diagnosis of PBL follows the criteria defined by Wiseman and Liao, according to which the patient must present with an appropriate specimen for diagnosis, have lymphocytic infiltrate and breast tissue in close proximity, show no evidence of concomitant systemic disease, and have no prior diagnosis of extramammary lymphoma 8 .

Imaging tests are very useful to aid in making the diagnosis, considering that most patients are asymptomatic. On mammography, it appears as a solitary, noncalcified mass in about $60 \%-70 \%$ of cases, whereas on ultrasound it does not present specific findings ${ }^{8}$.

After confirming the diagnosis of the tumor, having performed a histopathological analysis and an immunohistochemical study, other primary sites of the disease must be investigated to exclude the hypothesis of metastasis, with imaging tests such as MRI or CT. In the present case, the patient underwent PET-CT, a test that differs from conventional CT due to its properties of observing the metabolism of the site analyzed in the image, which did not locate additional lymphadenopathies ${ }^{11}$.

Disease staging is based on the Ann Harbor criteria, considering the involvement of lymph chains above the diaphragm and solid organs to define severity. Stages 1 and 2 define local diseases, as in the case in question, whereas stages 3 and 4 concern cases of systemically advanced diseases ${ }^{12}$.

Currently, there is no consensus in the literature regarding the best generalized therapeutic approach, as each case will depend on the biological behavior and histological characteristics of each lymphoma of the patients ${ }^{9,10}$.

For more indolent and localized lymphomas, radiotherapy alone or surgery are the best forms of intervention, with surgical treatment increasingly losing ground in the scientific field, as it does not have a higher survival rate or advantages in general when compared with radiotherapy. In these less aggressive cases, radiotherapy with a mean dose between 25-30 Gy has been adopted. Chemotherapy-based treatments have been preferred for cases of more aggressive and systemic tumors, and are deemed as the best choice both alone and in combination with other therapeutic approaches ${ }^{7-10}$.

The prognosis of patients affected by breast lymphomas will depend on their age, predictive factors, number of extranodal sites, course of treatment, and levels of LDH. It is estimated that the 5-year survival rate for patients with PBL is around 70\%?

\section{CONCLUSION}

PBL represents a rare type of breast tumor, with clinical features of other breast neoplasms. Although uncommon, its incidence has been growing in recent years, evidencing the importance of its inclusion in the differential diagnosis of breast cancer,

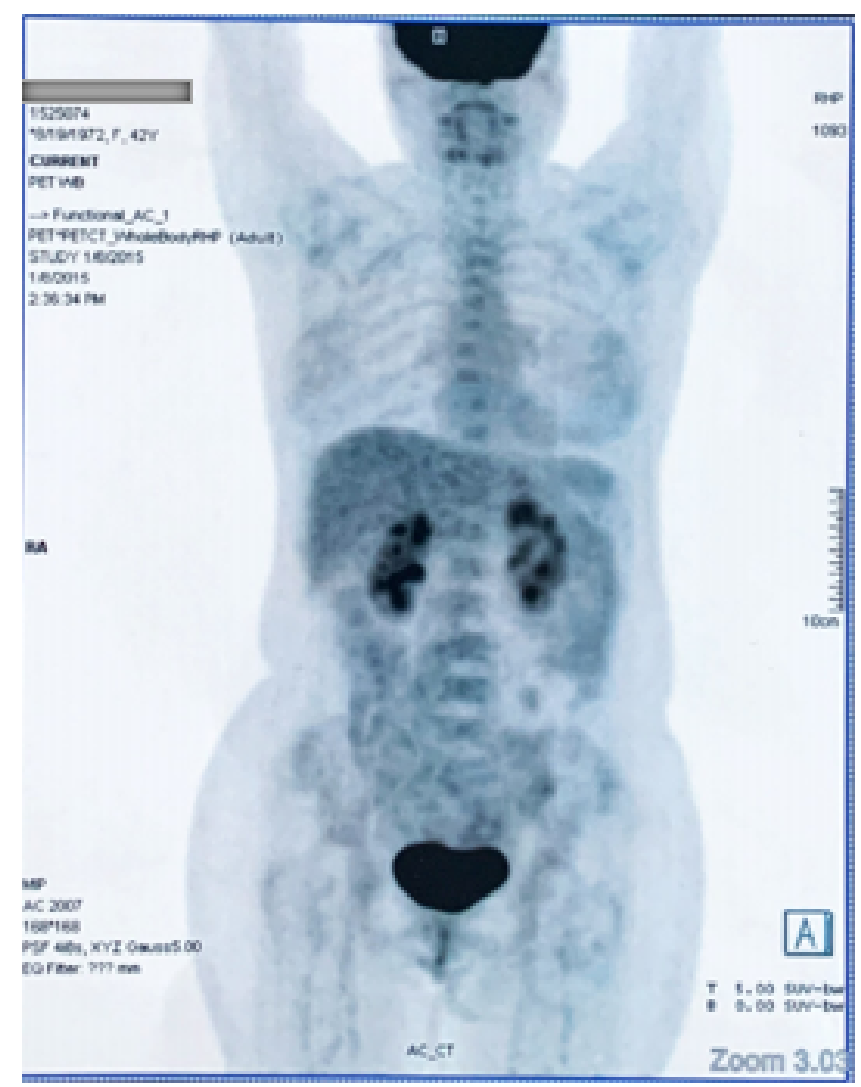

Figure 3. PET-CT coronal section, no additional mass or lymphadenopathy.

Table 1. Result of the immunohistochemical study.

\begin{tabular}{|c|c|c|c|}
\hline Antibodies & Clone & Result & Observation \\
\hline CD20 - B lymphocyte antigen & L26 & Positive & \\
\hline CD3 - T-cell receptor (epislon chain) & Polyclonal & Negative & \\
\hline $40,48,50$, and 50.6 kDa cytokeratins & AE1/AE3 & Positive & Epithelial cells \\
\hline Ki-67 (cell proliferation antigen) & M1B1 & Positive & $5 \%$, lymphoid cells \\
\hline CD10 - Common acute lymphoblastic leukemia antigen (CALLA) & $56 C 6$ & Positive & Follicular center cells \\
\hline Cyclin-D1 - cell cycle regulatory protein (bcl-1) & SP4 & Negative & \\
\hline
\end{tabular}


especially in the case of older patients. Considering that most cases are asymptomatic, the importance of breast exams and routine screening is emphasized.

\section{AUTHORS' CONTRIBUTION}

P.T.F.: Conceptualization, Data Curation, Investigation, Methodology, Project Administration, Writing — Original Draft, Writing - Review \& Editing.

S.H.P.F.: Conceptualization, Formal analysis, Project Administration, Supervision, Writing — Original Draft, Writing — Review \& Editing.
A.L.S.C.: Data Curation, Investigation, Methodology, Project Administration, Writing - Original Draft, Writing - Review \& Editing.

G.F.A.S.: Data Curation, Investigation, Methodology, Project Administration, Writing - Original Draft, Writing - Review \& Editing.

L.V.C.: Data Curation, Investigation, Methodology, Project Administration, Writing - Original Draft, Writing - Review \& Editing.

M.T.P.: Data Curation, Project Administration, Writing — Original Draft, Writing - Review \& Editing.

\section{REFERENCES}

1. Hissourou III M, Zia SY, Alqatari M, Strauchen J, Bakst RL. Primary MALT Lymphoma of the Breast Treated with Definitive Radiation. Case Rep Hematol. 2016;2016:1831792. http://doi.org/10.1155/2016/1831792

2. Aviv A, Tadmor T, Polliack A. Primary diffuse large B-cell lymphoma of the breast: looking at pathogenesis, clinical issues and therapeutic options. Ann Oncol. 2013;24(9):2236-44. http://doi.org/10.1093/annonc/mdt192

3. Zhang N, Cao C, Zhu Y, Liu P, Liu L, Lu K, et al. Primary breast lymphoma: A single center study. Oncol Lett. 2017;13(2):1014-8. http://doi.org/10.3892/ol.2016.5483

4. Joks M, Myśliwiec K, Lewandowski K. Primary breast lymphoma: a review of the literature and report of three cases. Arch Med Sci. 2011;7(1):27-33. http://doi.org/10.5114/ aoms.2011.20600

5. Cheah CY, Campbell BA, Seymour JF. Primary breast lymphoma. Cancer Treat Rev. 2014;40(8):900-8. http://doi. org/10.1016/j.ctrv.2014.05.010

6. Shao JM, Cheney M, Oppong BA. Primary mucosa associated lymphoid tissue (MALT) B-cell lymphoma - a rare breast malignancy. Ame Med J. 2016;1(3):5. http://doi.org/10.21037/ amj.2016.12.01
7. Rock K, Rangaswamy G, O’Sullivan S, Coffey J. An unusual case of marginal zone b-cell lymphoma arising in the breast: its diagnosis and the role of radiotherapy in its management. Breast Care (Basel). 2011;6(5):391-3. http://doi. org/10.1159/000333128

8. Radkani P, Joshi D, Paramo JC, Mesko TW. Primary breast lymphoma:30years of experience with diagnosis and treatment at a single medical center. JAMA Surg. 2014;149(1):91-3. http:// doi.org/10.1001/jamasurg.2013.2283

9. Koganti SB, Lozada A, Curras E, Shah A. Marginal zone lymphoma of the breast-A diminished role for surgery. Int J Surg Case Rep. 2016;25:4-6. http://doi.org/10.1016/j.ijscr.2016.05.041

10. Foo MY, Lee WP, Seah CMJ, Kam C, Tan SM. Primary breast lymphoma: a single-centre experience. Cancer Rep. 2019;2:e1140. http://doi.org/10.1002/cnr2.1140

11. Gonçalves JTV, Giordani RR, Lima PL, Rangel KK, Melo GL, Paim SP, et al. Linfoma primário de mama: relato de caso. Rev Bras Mastologia. 2011;21(4):178-80.

12. Avenia N, Sanguinetti A, Cirocchi R, Bistoni G, Trastulli S, D'Ajello F, et al. Primary breast lymphomas: a multicentric experience. World J Surg Oncol. 2010;8:53. http://doi. org/10.1186/1477-7819-8-53 